\title{
Mid-long-term results of total knee arthroplasty followed by ipsilateral total hip arthroplasty versus total hip arthroplasty subsequent to ipsilateral total knee arthroplasty: A case-control analysis
}

\section{Zunhan Liu}

West China Hospital

Wei-Nan Zeng

West China Hospital

Zhenyu Luo

Sichuan University West China Hospital

Enze Zhao

Sichuan University West China Hospital

Hao Li

Sichuan University West China Hospital

Zongke Zhou ( $\nabla$ zhouzongke@scu.edu.cn )

Sichuan University West China Hospital https://orcid.org/0000-0002-9037-4756

Research article

Keywords: Total knee arthroplasty, Total hip arthroplasty, Ipsilateral, Mechanical axis, Valgus

Posted Date: January 19th, 2021

DOI: https://doi.org/10.21203/rs.3.rs-52537/v2

License: (c) (1) This work is licensed under a Creative Commons Attribution 4.0 International License.

Read Full License 


\section{Abstract}

Background: The aim of the present study was to compare the outcomes of patients who underwent different sequences of ipsilateral total hip arthroplasty (THA) and total knee arthroplasty (TKA).

Methods: We retrospectively identified 47 patients who underwent TKA followed by ipsilateral THA (THATKA) and 36 patients who received THA subsequent to ipsilateral TKA (TKA-THA) for rheumatoid arthritis or osteoarthritis between January 2008 and April 2014. Twenty-eight patients were selected for each group after case-control matching with preoperative demographics and protheses of THA. Clinical scores, radiographic results, complication rates, and survivorship were compared. The median duration of followup was 110 (range 80-149) months.

Results: Both groups showed significant improvement in Harris Hip Scores, Knee Society Score, and Short Form-12 at the last follow-up compared to baseline $(p<.001)$. At the last follow-up, all clinical scores were actually lower in the THA-TKA group, but those differences were not statistically significant. Otherwise, there was no significant difference in radiological alignment or complication rates. The survivorship of THA and TKA in the THA-TKA group was $88.2 \%$ and $87.4 \%$, respectively, compared with $75.0 \%$ and $95.7 \%$ in the TKA-THA group at 10 years (log rank, $p=.939$ and .187 ).

Conclusions: Patients who underwent ipsilateral THA and TKA with different sequences achieved similar favorable outcomes. Total joint arthroplasty can be performed safely with excellent outcomes in patients with a history of prior ipsilateral THA or TKA.

\section{Background}

Although the utilization of disease-modifying medications has improved the quality of life of patients with symptomatic arthritis, the number of people affected with multiple lower extremity joints remains high $[1,2]$. Subsequently, the possibility of performing total joint arthroplasty (TJA) on the ipsilateral hip and knee in the same patient may increase. A previous study reported that the cumulative incidences of total knee arthroplasty (TKA) followed by ipsilateral total hip arthroplasty (THA) and THA subsequent to ipsilateral TKA were $2.1 \%$ and $1.8 \%$, respectively, at the 20-year follow-up [3].

TJA is one of the most successful health care interventions. Patients with end-stage degenerative or inflammatory arthritis report excellent outcomes after THA or TKA [4]. However, there is some evidence to suggest loading redistribution and biomechanical changes in the lower limb after THA or TKA [5-9]. Data concerning the influence of prior THA on the ipsilateral knee joint or ipsilateral TKA remain controversial. Several studies have reported that the lateral patellar tilt increased and the external knee adduction moment decreased after ipsilateral THA, which may lead to abnormal loading on the knees and ipsilateral knee pain $[7,5,8]$. Other studies have suggested that there is no increase in biomechanical loading during gait and posture on ipsilateral or contralateral knee joints after unilateral THA [10]. Furthermore, in certain cases, it is difficult to thoroughly insert the femoral intramedullary guide into the femoral canal during ipsilateral TKA due to a prior femoral prosthesis, increasing the chance of malalignment [11]. To 
date, only one study has analyzed the influence of prior THA on the functional outcome of subsequent ipsilateral TKA and demonstrated the influence is limited [12]. On the other hand, the influence of a prior TKA on the ipsilateral hip has also been examined. Previous studies have reported that prior TKA leads to biomechanical changes and that knee alignment caused by prior TKA affects the positioning of the ipsilateral femoral component during THA [6,13-15]. However, to our knowledge, no study has yet compared the clinical outcomes and implant survivorship in patients undergoing different sequences of ipsilateral THA and TKA.

Therefore, the primary purpose of this study was to determine whether the different sequences of ipsilateral THA and TKA would have different clinical scores. As a secondary outcome, we examined whether the different sequences of ipsilateral THA and TKA would lead to different radiological alignments or component positions, complication rates, and implant survivorship. We hypothesized that patients undergoing different sequences of ipsilateral THA and TKA would have similar clinical outcomes, complication rates, and implant survivorship at the last follow-up.

\section{Methods}

\section{Patients}

This retrospective study was approved by the Institutional Review Board of West China Hospital (ID: 2012-268) and was reported in accordance with the STROCSS criteria [16]. The inclusion criteria were patients with severe pain and/or considerable difficulty in performing daily activities refractory to nonoperative management who underwent ipsilateral THA and TKA for rheumatoid arthritis (RA) or osteoarthritis (OA). The exclusion criteria were hip dysplasia, acetabulum or femoral fracture, ankylosing spondylitis, prior lower extremity fracture, posttraumatic arthritis, revision THA or TKA, and incomplete clinical or radiographic records. Additionally, to avoid bias related to prior TJA, patients with an interval time between ipsilateral THA and TKA shorter than 6 months and patients who underwent prior TJA with a functional score at the time of subsequent surgery less than 70 points were also excluded. We identified 88 patients (97 hips and 97 knees) who underwent ipsilateral THA and TKA at our institution between January 2008 and April 2014. Of these, two patients (2 hips and 2 knees) were lost to follow-up and could not be contacted via telephone, while three patients ( 4 hips and 4 knees) died from diseases unrelated to the operation. According to different sequences of ipsilateral THA and TKA, the remaining 83 patients (91 hips and 91 knees), including 47 patients undergoing TKA followed by ipsilateral THA and 36 as THA subsequent to ipsilateral TKA, were classified into 2 groups. To minimize possible confounding factors, the two groups were statistically matched for age (up to \pm 10 years), sex, cause of TJA, primary surgery data (up to \pm 12 months), and THA prosthesis. Because all implants of primary TKA in our center were posterior-stabilized, we did not match the prosthesis of TKA. Ultimately, twenty-eight patients were selected for each group and included in the final analysis.

\section{Surgical techniques}


All THA procedures were performed using a posterolateral approach under general anesthesia. The cementless porous-coat acetabular fixations (Pinnacle implants, DePuy Orthopaedics) were routinely press-fitted into the acetabulum at $15 \pm 10^{\circ}$ of anteversion and $40 \pm 10^{\circ}$ of inclination. If necessary, supplemental screws were used to achieve implant stability. Two cementless femoral stems, Corail and Summit (DePuy Synthes, Warsaw, IN), were inserted into the hips. Of these, ceramic-on-ceramic articulations were utilized in 42 hips (75.0\%), and ceramic-on-polyethylene was used in 14 hips (25.0\%).

For the ipsilateral TKA procedure, the knee was exposed by a standard medial parapatellar approach, and osteophytes, worn meniscus, and posterior cruciate ligament of the knee were resected. After determining the entry point, we inserted a femoral intramedullary alignment rod in the center of the femoral intercondylar notch with the distal femoral cutting guide set for individual degrees measured before surgery. Of note, one knee in the THA-TKA group and 2 knees in the TKA-THA group had a preoperative anatomic valgus of $>20^{\circ}$ that we released the iliotibial band (ITB) using the "pie-crusting" technique [17]. If the lateral tension on extension and flexion was still tight, the posterlateral capsule was also released, avoiding the collateral ligament (LCL) and popliteus tendon (POP). After assessing the extension gap and balancing the flexion gap, we performed cemented, posterior-stabilized TKAs on all subjects with two total knee implants (DePuy Sigma PFC and Stryker Scorpio NRG). All patients received intraoperative and postoperative prophylactic broad-spectrum antibiotics and tranexamic acid antithrombotic therapy. Postoperatively, active flexion-extension ankle motion and quadriceps strengthening exercises were encouraged. Partial weight-bearing with crutches as tolerated on the second postoperative day and full weight-bearing were allowed from the third day. For patients with THA, simultaneous flexion and internal rotation were avoided after surgery.

\section{Clinical evaluations and radiographic assessments}

Clinical follow-up was conducted routinely at 3 weeks, 3 months and 6 months after the procedures and annually thereafter until the final follow-up. The clinical evaluation protocol included the Harris Hip Score (HHS) [18], Knee Society Score (KSS) [19], and Short Form-12 scale (SF-12) [20]. Radiographs (serial standardized anteroposterior and lateral radiographs of hip and knee, and full-length weight-bearing anteroposterior films) were taken for patients preoperatively and performed at each follow-up. To minimize the variability of the interobserver, all radiographic measurements were performed independently and averaged by 2 trained investigators in the index surgery. To describe the coronal extremity axis, the hip-knee-ankle (HKA) angle was defined as the medial angle between the mechanical axis of the femur and the mechanical axis of the tibia. The femorotibial angle (FTA) was defined as the lateral angle between the anatomical axis of the femur and the anatomical axis of the tibia. When assessing coronal alignment on full-length films, an HKA less than $177^{\circ}$ was considered varus, neutral between $177^{\circ}$ and $183^{\circ}$, and valgus greater than $183^{\circ}$. Of note, severe valgus knee alignment was defined as an FTA of $<160^{\circ}$ [21]. The femoral offset (FO) was defined as the perpendicular distance from the centerline of the proximal part of the femoral shaft to the center of the femoral head. The inclination angle was defined as the angle formed by the inter-teardrop line and the long axis of the cup opening ellipse. The limb length discrepancy (LLD) was calculated by comparing the vertical distance from the 
prominent points of the lesser trochanter to the teardrop line. If the deformation of the lesser trochanter was severe, we used the tip of the greater trochanter as the reference. The correction in LLD was defined as the height difference in the preoperative and postoperative LLD [22] (Fig. 1).

Serial radiographs were also evaluated for evidence of postoperative periprosthetic fracture (PFF), dislocation, subsidence, and femoral component stability. Subsidence of the femoral component was defined as the change in the distance from the center of the femoral head to the lesser trochanter by the method of Heekin et al [23]. The femoral component stability was evaluated and graded as bone stable, fibrous stable, or unstable, according to the criteria described by Engh et al [24]. Complications included periprosthetic infection, deep venous thrombosis, and neurologic injury. Kaplan-Meier survivorship analysis was performed on all THAs and TKAs using a standard case scenario where all arthroplasties were considered to be successful at the final follow-up. Prosthesis failure was defined as any reason for aseptic revision.

\section{Statistical analysis}

Groups of THA-TKA and TKA-THA were matched one to one using case-control matching, and statistical analyses were performed using SPSS statistical software, version 25.0 (IBM Corp., Armonk, NY). The figures were generated by GraphPad Prism version 8.0. The students' t-test or Mann-Whitney U-test was used to analyze continuous variables between two groups. Pearson's chi-squared test or Fisher's exact test was used for discontinuous variables between two groups. Implant survivorships were analyzed by Kaplan-Meier curves with revision for any reason other than infection as the endpoint. The survival rate between the 2 groups was compared by the log-rank test. $a=.05, P<.05$ indicated statistical significance.

\section{Results}

The preoperative and postoperative clinical scores and radiological results are summarized in Table 2. No significant difference was detected in preoperative clinical parameters, including HHS, KSS, and SF-12. In both groups, the mean HHS, KSS, and SF-12 score significantly improved from preoperatively to the last follow-up $(p<.001)$. At the last clinical evaluation, the postoperative parameters, including HHS, KSSknee score and function score, physical component summary (PCS) and mental component summary (MCS) of SF-12, were actually lower in the THA-TKA group. However, those differences were not significant $(p>.05)$.

There was also no significant difference in preoperative radiographic measurements between the 2 groups, including HKA, KAA, FO, and LLD (Table 2). However, preoperative mean HKA and FTA indicated valgus alignment in both groups $\left(184.1^{\circ}\right.$ vs $184.1^{\circ}, 173.0^{\circ}$ vs $\left.173.2^{\circ}, p>.05\right)$. The comparisons of lower limb alignment parameters were based on the measured data of the full-length weight-bearing radiographs. Relative to the mechanical and anatomical alignment, HKA and FTA were corrected to neutral after surgery $(p<.001)$. The postoperative FO, LLD, and cup inclination did not differ between the 2 groups $(p=.967, .137, .323)$. 
No periprosthetic infection or deep venous thrombosis was identified during the follow-up in each group (Table 3). There was 1 case of sciatic nerve palsy patient in the TKA-THA group after THA who recovered spontaneously within 9 months without residual symptoms. Intraoperative fracture occurred in 4 hips (1 in the distal femur and 3 in the proximal femur) and 1 knee without displaced cracks or perforation; all the fractures were treated with immediate cerclage wire fixation. Two patients ( 2 hips) experienced postoperative dislocation in the THA-TKA group who were treated with closed manipulative reduction and confined to bed for 4 weeks. Two patients in the THA-TKA group and 1 patient in the TKA-THA group had an over $5 \mathrm{~mm}$ subsidence of the femoral stem and underwent stem revisions with subsequent stabilization and evidence of fibrous stability at the last follow-up. Of the patients with aseptic revisions, in the THA-TKA group, two femoral revisions were performed for stem subsidence, and one acetabular revision was performed for recurrent dislocation. For ipsilateral TKA, two knee revisions were performed for tibial aseptic loosening and 1 was performed for instability. In contrast, in the TKA-THA group, one femoral stem revision was performed for subsidence, one modular liner was changed because of dissociation of the highly cross-linked polyethylene insert from the outer shell, and one knee revision was performed due to tibial implant loosening. There was no difference between the THA-TKA group and the TKA-THA group with respect to the complication rate and overall revisions $(p>.05)$. Considering aseptic revision as an endpoint (Fig. 2), THA survival at 10 years was $88.2 \%$ (95\% confidence interval $75.7-99.9 \%)$ and $87.4 \%$ (95\% confidence interval 70.9-99.9\%) in the THA-TKA group and TKA-THA group, respectively (log-rank, $p=.939)$. In contrast, the TKA survival at 10 years was $75.0 \%$ (95\% confidence interval 49.999.9\%) and $95.7 \%$ (95\% confidence interval $87.2-99.9 \%)$ in the THA-TKA group and TKA-THA group at 10 years, respectively (log-rank, $p=.187)$.

\section{Discussion}

Previous studies demonstrated that biomechanical changes and loading redistribution after prior THA or TKA may affect the ipsilateral hip or knee [5-9]. However, no study has compared the clinical outcomes and implant survivorship in patients underwent who different sequences of ipsilateral THA and TKA. Our primary hypothesis was confirmed; the main finding of this study was that there was no significant difference between the 2 groups in HHS, KSS, and SF-12 score at the last follow-up.

Only one study [12] assessed the influence of a prior THA on axial alignment and the clinical outcome of a subsequent ipsilateral TKA by comparing patients who underwent TKA followed by ipsilateral THA and those who only underwent TKA. Compared with the most comparable study by Asensio-Pascual et al [12], the clinical scores from our results were lower than those reported in their study, with mean postoperative HHS, KSS-knee, and KSS-function score of averaged 86.4, 87.6, 88.3, respectively in the THA-TKA group. A possible interpretation is that disease of our study, including RA, which is involved in multiple joint sites, including metatarsal phalangeal joints and the ankle, might be present even after TJA. Although RA is a rare indication for TJA compared to $\mathrm{OA}$, it has been the most common cause of widespread involvement of the multiple lower extremity joints [25-27]. The immobility of other lower limb joints could lead to difficulty ambulating or ascending stairs despite evident improvements in hip and knee function. Several 
previous studies involving RA patients primarily for multiple joint arthroplasties reported that between $46.2 \%$ and $62.5 \%$ of patients required walking aids, and the ability to walk was limited $[25,28,29]$.

Comparison of the radiological results and complication rate with the only study [12] is difficult because it evaluated lower limb alignment without full-length weight-bearing radiographs and did not systematically report the results of complications. Patients undergoing ipsilateral hip and knee surgeries presented preoperative valgus deformity in the current study. Preoperative knee valgus deformity was associated with advanced RA, which was in agreement with a previous study [30]. Preoperative valgus deformity means that the procedure of TKA is much more technically challenging, including obtaining a proper component rotational alignment and balancing soft tissue in both flexion and extension with the least constraint. Although knees with preoperative valgus were corrected to neutral and no significant differences were detected between the 2 groups in axial alignment at the last follow-up, our overall implant survivorship of TKA in the THA-TKA group was lower than that reported in Asensio-Pascual et al [12], with $96.6 \%$ survivorship at 7.2 years. It may be that preoperative valgus alignment failed at a higher rate than preoperatively neutral knees even when corrected to neutral postoperative alignment [2830]. Another possible reason may be that survivorship after TJA seems to be lower in patients with RA than in those with OA [31].

In clinical practice, if the ipsilateral hip and knee simultaneously meet the indications for TJA, most surgeons would suggest performing THA before TKA. Active flexion and extension of the knee depend largely on free hip function and knee pain is always associated with ipsilateral hip dysfunction. However, certain affected multiple joint diseases, such as RA, normally erode the knees first, leading to compulsory TKA. Then, the disease severity of the ipsilateral hip gradually developed to a degree that met the indication for THA. We agreed with the idea that the sequence of arthroplasties should depend on the severity of symptoms and that the most symptomatic joint of the hip or knee should be replaced first $[8,32]$.

Although biomechanical changes and redistribution of loading of the lower limb were detected after THA or TKA in certain studies [5-9], the influence of a prior TJA on the subsequent ipsilateral THA or TKA is limited. Consistent with the findings of the current study, Foucher et al [10] reported no increase in biomechanical loading during gait on the ipsilateral knee after THA. Likewise, other studies demonstrated that the changes in the axial alignment of the lower extremity after THA could result in an increased overload on the contralateral knee rather than ipsilateral knee which was characterized by compensation to minimize the loading of the affected limb [7]. Thus, patients who undergo THA may have higher risks of developing $\mathrm{OA}$ in the contralateral knee than in the ipsilateral knee [33].

To date, this is the first study to compare the mid to long-term results of patients who underwent different sequences of ipsilateral THA and TKA. The strengths of this study include the completeness of clinical and radiographic data, uncemented hip prostheses and posterior-stabilized knee prostheses, and the homogeneity of the surgical technique. However, we note that there are several limitations as well. First, the retrospective data and a relatively small sample population for each group decreased the level of 
evidence. Second, only coronal alignment was evaluated in present study, although the rotational alignment and patellar tilt are also important for the success of TKA. However, this study focused on modification of the mechanical axis, which is mainly present in coronal alignment. Future research with three-dimensional CT would be useful in studying modifications in rotational alignment.

\section{Conclusion}

Patients undergoing different ipsilateral THA and TKA sequences could achieve similar favorable clinical outcomes. Total joint arthroplasty can be performed safely with excellent outcomes in patients with a history of prior ipsilateral THA or TKA.

\section{List Of Abbreviations}

TJA, total joint arthroplasty; THA, total hip arthroplasty; TKA, total knee arthroplasty; RA, rheumatoid arthritis; OA, osteoarthritis; HKA, hip-knee-angle; ITB, iliotibial band; LCL, collateral ligament; POP, popliteus tendon; HHS, Harris Hip Score; KSS, Knee Society Score; SF-12, Short Form-12 Score; FTA; femorotibial angle; FO, femoral offset; LLD, limb length discrepancy; PFF, periprosthetic fracture; PCS, physical component summary; MCS, mental component summary.

\section{Declarations}

\section{Acknowledgement}

We would like to thank the relevant staff for guidance and assistance for their support and collaboration in our hospital.

\section{Funding}

This research did not receive any specific grant from funding agencies in the public, commercial, or notfor-profit sectors.

\section{Availability of data and materials}

The datasets used or analyzed in the current study are available from the corresponding author up reasonable request.

\section{Author contributions}

Zunhan Liu, Wei-Nan Zeng, and Zongke Zhou participated in the design and coordination of the study, collected the data, analyzed the data, and drafted the manuscript. Zhenyu Luo, Enze Zhao, and Hao Li assisted in collecting the data and drafting the manuscript. All authors have read and approved the final manuscript. 


\section{Ethics approval and consent to participate}

This study was approved by the Ethics Committee and Institutional Review Board of West China Hospital, Sichuan University (2012-268). Informed consent was obtained from each patient.

\section{Consent for publication}

All patients provided consent to participate in the current study, per our institution's standard for research consent forms. This consent specified that the research information obtained may be used for publication.

\section{Competing interests}

The corresponding author Dr. Zongke Zhou is a member of the editorial board of BMC Musculoskeletal Disorders, and the rest of the authors declare they have no competing interests.

\section{Publisher's note}

Springer Nature remains neutral with regard to jurisdictional claims in published maps and institutional affiliations.

\section{Additional information}

The authors declare that they have no competing financial interests.

\section{References}

1. Mertelsmann-Voss C, Lyman S, Pan TJ, et al. US trends in rates of arthroplasty for inflammatory arthritis including rheumatoid arthritis, juvenile idiopathic arthritis, and spondyloarthritis. Arthritis Rheumatol. 2014;66 (6):1432-1439.

2. Ravi B, Croxford R, Reichmann WM, et al. The changing demographics of total joint arthroplasty recipients in the United States and Ontario from 2001 to 2007. Best Pract Res Clin Rheumatol. 2012;26 (5):637-647.

3. Sanders TL, Maradit Kremers H, Schleck CD, et al. Subsequent Total Joint Arthroplasty After Primary Total Knee or Hip Arthroplasty: A 40-Year Population-Based Study. J Bone Joint Surg Am. 2017;99 (5):396-401.

4. Learmonth ID, Young C, Rorabeck C. The operation of the century: total hip replacement. Lancet. 2007;370 (9597):1508-1519.

5. Akiyama K, Nakata K, Kitada M, et al. Chronological Changes in Axial Alignment of the Ipsilateral Hip and Knee After Total Hip Arthroplasty. J Arthroplasty. 2018;33 (2):415-422.

6. Nam KW, Tsai TY, Dimitriou D, et al. Ipsilateral varus knee alignment correlates with increased femoral stem anteversion in primary total hip arthroplasty. Hip Int. 2016;26 (2):175-179. 
7. Akiyama K, Nakata K, Kitada M, et al. Changes in axial alignment of the ipsilateral hip and knee after total hip arthroplasty. Bone Joint J. 2016;98-b (3):349-358.

8. 8. Stief F, Schmidt A, van Drongelen $S$, et al. Abnormal loading of the hip and knee joints in unilateral hip osteoarthritis persists two years after total hip replacement. J Orthop Res. 2018.

9. Wang W, Geller JA, Nyce JD, et al. Does ipsilateral knee pain improve after hip arthroplasty? Clin Orthop Relat Res. 2012;470 (2):578-583.

10. Foucher KC, Wimmer MA. Contralateral hip and knee gait biomechanics are unchanged by total hip replacement for unilateral hip osteoarthritis. Gait Posture. 2012;35 (1):61-65.

11. Webb BT, Ulrich SD, MacKinlay KGW, et al. Use of Shorter Intramedullary Guide for Ipsilateral Total Knee Arthroplasty following Prior Total Hip Arthroplasty. J Knee Surg. 2018;31 (4):348-351.

12. Asensio-Pascual A, Lizaur-Utrilla A, Vizcaya-Moreno MF, et al. Prior unilateral total hip arthroplasty does not influence the outcome of ipsilateral total knee arthroplasty. Knee Surg Sports Traumatol Arthrosc. 2019;28(5):1452-1457.

13. Alnahdi AH, Zeni JA, Snyder-Mackler L. Gait after unilateral total knee arthroplasty: frontal plane analysis. J Orthop Res. 2011;29 (5):647-652.

14. Milner CE, O'Bryan ME. Bilateral frontal plane mechanics after unilateral total knee arthroplasty. Arch Phys Med Rehabil. 2008;89 (10):1965-1969.

15. Worsley $P$, Stokes $M$, Barrett $D$, et al. Joint loading asymmetries in knee replacement patients observed both pre- and six months post-operation. Clin Biomech (Bristol, Avon). 2013;28 (8):892-897.

16. Agha R, Abdall-Razak A, Crossley E, et al. STROCSS 2019 Guideline: Strengthening the reporting of cohort studies in surgery. Int J Surg. 2019;72:156-165.

17. Ranawat AS, Ranawat CS, Elkus $M$, et al. Total knee arthroplasty for severe valgus deformity. J Bone Joint Surg Am. 2005;87 Suppl 1 (Pt 2):271-284.

18. Harris WH. Traumatic arthritis of the hip after dislocation and acetabular fractures: treatment by mold arthroplasty. An end-result study using a new method of result evaluation. J Bone Joint Surg Am. 1969;51 (4):737-755.

19. Insall JN, Dorr LD, Scott RD, et al. Rationale of the Knee Society clinical rating system. Clin Orthop Relat Res. 1989;(248):13-14.

20. Ware J, Jr., Kosinski M, Keller SD. A 12-Item Short-Form Health Survey: construction of scales and preliminary tests of reliability and validity. Med Care. 1996;34 (3):220-233.

21. Liu HX, Shang $P$, Ying $X Z$, et al. Shorter survival rate in varus-aligned knees after total knee arthroplasty. Knee Surg Sports Traumatol Arthrosc. 2016;24 (8):2663-2671.

22. Takao M, Ohzono K, Nishii T, et al. Cementless modular total hip arthroplasty with subtrochanteric shortening osteotomy for hips with developmental dysplasia. J Bone Joint Surg Am. 2011;93 (6):548-555.

23. Heekin RD, Engh CA, Herzwurm PJ. Fractures through cystic lesions of the greater trochanter. A cause of late pain after cementless total hip arthroplasty. J Arthroplasty. 1996;11 (6):757-760. 
24. Engh CA, Massin P, Suthers KE. Roentgenographic assessment of the biologic fixation of poroussurfaced femoral components. Clin Orthop Relat Res. 1990;(257):107-128.

25. Hoekstra HJ, Veth RP, Nielsen HK, et al. Bilateral total hip and knee replacement in rheumatoid arthritis patients. Arch Orthop Trauma Surg. 1989;108 (5):291-295.

26. McDonald I. Bilateral replacement of the hip and knee in rheumatoid arthritis. J Bone Joint Surg Br. 1982;64 (4):465-468.

27. Meding JB, Faris PM, Davis KE. Bilateral Total Hip and Knee Arthroplasties: Average 10-Year FollowUp. J Arthroplasty. 2017;32 (11):3328-3332.

28. Jergesen HE, Poss R, Sledge CB. Bilateral total hip and knee replacement in adults with rheumatoid arthritis: an evaluation of function. Clin Orthop Relat Res. 1978;(137):120-128.

29. Johnson KA. Arthroplasty of both hips and both knees in rheumatoid arthritis. J Bone Joint Surg Am. 1975;57 (7):901-904.

30. Tucker A, O'Brien S, Doran E, et al. Total Knee Arthroplasty in Severe Valgus Deformity Using a Modified Technique-A 10-Year Follow-Up Study. J Arthroplasty. 2019;34 (1):40-46.e41.

31. Feng $B$, Weng $X$, Lin J, et al. Long-term follow-up of cemented fixed-bearing total knee arthroplasty in a Chinese population: a survival analysis of more than 10 years. J Arthroplasty. 2013;28 (10):17011706.

32. Strand V, Singh JA. Improved health-related quality of life with effective disease-modifying antirheumatic drugs: evidence from randomized controlled trials. Am J Manag Care. 2008;14 (4):234254

33. Umeda N, Miki H, Nishii T, et al. Progression of osteoarthritis of the knee after unilateral total hip arthroplasty: minimum 10-year follow-up study. Arch Orthop Trauma Surg. 2009;129 (2):149-154.

\section{Tables}

\section{Table 1}

Baseline characteristics of the 2 case control groups.

\begin{tabular}{llll}
\hline Parameters & $\begin{array}{l}\text { THA-TKA group } \\
\mathrm{n}=28\end{array}$ & $\begin{array}{l}\text { TKA-THA group } \\
\mathrm{n}=28\end{array}$ & $p$ value \\
\hline Gender (male/female). & $3 / 25$ & $3 / 25$ & $1.000^{\mathrm{b}}$ \\
Age (yr) & $57.3 \pm 10.6$ & $58.8 \pm 10.2$ & $0.610^{\mathrm{a}}$ \\
Body mass index $\left(\mathrm{kg} / \mathrm{m}^{2}\right)$ & $22.3 \pm 2.5$ & $22.7 \pm 3.9$ & $0.664^{\mathrm{a}}$ \\
ASA (I-II/III-IV) & $19 / 9$ & $22 / 6$ & $0.365^{\mathrm{c}}$ \\
No. of diseases (RA/OA) & $17 / 11$ & $17 / 11$ & $1.000^{\mathrm{b}}$ \\
Varus/neutral/valgus/severe valgus & $2 / 11 / 14 / 1$ & $3 / 6 / 17 / 2$ & $0.514^{\mathrm{b}}$ \\
THA follow-up (mo) & $109(80$ to 142$)$ & $97.5(68$ to140) & $0.070^{\mathrm{a}}$ \\
TKA follow-up (mo) & $100(72$ to 131$)$ & $114.5(85$ to 149$)$ & $0.003^{\mathrm{a}}$ \\
Interval between surgery (mo) & $11(6$ to 21$)$ & $14(6$ to 23$)$ & $0.187^{\mathrm{a}}$ \\
\hline
\end{tabular}

ASA, American Society of Anesthesiologists Scale; RA, rheumatoid arthritis; OA, osteoarthritis. a Student $t$ test.

${ }^{b}$ Pearson chi-squared or Fisher's exact test. 
c Mann-Whitney Utest.

Table 2 Implant informations.

\begin{tabular}{llll}
\hline Characteristics & THA-TKA group & TKA-THA group & $p$ value \\
\hline Femoral stem & & & \\
Corial & 21 & 20 & 0.783 \\
\hline Summit & 5 & 7 & 0.522 \\
\hline Tri-Lock & 3 & 2 & 0.643 \\
\hline S-ROM & 1 & 1 & 1.000 \\
\hline Ceramic-on-ceramic & 22 & 21 & 0.776 \\
\hline Ceramic-on-polythylene & 8 & 9 & 0.776 \\
\hline Total knee implants & & & \\
\hline Johnson \& Johnson/DePuy & 13 & 14 & 0.797 \\
\hline Stryker & 15 & 15 & 1.000 \\
\hline TC3 & 2 & 1 & 0.557 \\
\hline
\end{tabular}

The Corail, Summit, Tri-Lock, and S-ROM prostheses are manufactured by Depy (DePuy, Warsaw, IN).

Table 3

Preoperative and postoperative clinical outcomes.

\begin{tabular}{llll}
\hline Parameters & $\begin{array}{l}\text { THA-TKA group } \\
\mathrm{n}=28\end{array}$ & $\begin{array}{l}\text { TKA-THA group } \\
\mathrm{n}=28\end{array}$ & $p$ value \\
\hline Harris Hip Score & $34.0 \pm 4.3$ & $34.5 \pm 6.0$ & 0.722 \\
$\quad$ Preoperative & $82.2 \pm 6.4$ & $83.4 \pm 6.9$ & 0.496 \\
\hline Last follow-up & & & \\
\hline Knee Society Score & $32.4 \pm 6.4$ & $32.4 \pm 8.6$ & 0.986 \\
\hline Preoperative knee score & $86.1 \pm 6.3$ & $86.4 \pm 8.4$ & 0.901 \\
\hline Last follow-up knee score & $38.0 \pm 6.0$ & $37.0 \pm 6.0$ & 0.506 \\
\hline Preoperative function score & $85.0 \pm 4.3$ & $86.1 \pm 4.8$ & 0.382 \\
\hline Last follow-up function score & 85.0 & \\
\hline SF-12 & $10.5 \pm 2.4$ & $10.6 \pm 2.6$ & 0.831 \\
\hline Preoperative PCS & $20.8 \pm 2.2$ & $21.0 \pm 2.1$ & 0.804 \\
\hline Last follow-up PCS & $13.3 \pm 2.5$ & $13.5 \pm 2.9$ & 0.730 \\
\hline Preoperative MCS & $22.9 \pm 1.8$ & $23.1 \pm 1.9$ & 0.519 \\
\hline Last follow-up MCS & & & \\
\hline HKA ( ${ }^{\circ}$ ) & $184.1 \pm 5.3$ & $184.1 \pm 5.5$ & 0.496 \\
\hline Preoperative & $179.7 \pm 3.3$ & $179.6 \pm 2.4$ & 0.645 \\
\hline Last follow-up & & & \\
\hline FTA $\left(^{\circ}\right.$ ) & $173.0 \pm 5.5$ & $173.2 \pm 5.8$ & 0.600 \\
\hline Preoperative & $177.7 \pm 3.2$ & $177.6 \pm 2.3$ & 0.178 \\
\hline Last follow-up & & & \\
\hline FO & $39.0 \pm 9.0$ & $39.8 \pm 7.9$ & 0.705 \\
\hline Preoperative & $40.5 \pm 6.5$ & $40.4 \pm 9.5$ & 0.967 \\
\hline Last follow-up & $37.8 \pm 11.7$ & $41.2 \pm 8.1$ & 0.149 \\
\hline LLD & $43.0 \pm 8.0$ & $46.5 \pm 9.1$ & 0.137 \\
\hline Preoperative & $41.9 \pm 5.9$ & $40.3 \pm 6.2$ & 0.323 \\
\hline Last follow-up & & & \\
\hline Cup inclination $\left({ }^{\circ}\right)$ & & & \\
\hline
\end{tabular}

PCS, physical component summary; MCS, mental component summary; HKA, hip knee ankle angle; FTA, femorotibial angle; FO, femoral offset; LLD, limb length discrepancy.

The values are present as the mean \pm SD.

\section{Table 4}

Complications and Revisions 


\begin{tabular}{lccc}
\hline Complications & $\begin{array}{c}\text { THA-TKA group } \\
\mathrm{n}=28\end{array}$ & $\begin{array}{c}\text { TKA-THA group } \\
\mathrm{n}=28\end{array}$ & $p$ value \\
\hline Periprosthetic infection & 0 & 0 & 1.000 \\
Deep venous thrombosis & 0 & 0 & 1.000 \\
Sciatic nerve palsy & 0 & 1 & 1.000 \\
Postoperative PFF & 4 & 2 & 0.669 \\
Early postoperative dislocation & 2 & 0 & 0.154 \\
Femoral component stability & 22 & 25 & 0.429 \\
Bony stable & 5 & 3 & \\
Fibrous stable & 1 & 0 & \\
Unstable & 2 & 1 & 1.000 \\
Femoral stem subsidence & 3 & 2 & 0.939 \\
Revision & 3 & 1 & 0.187 \\
Total hip revision & 3 & & \\
Total knee revision & & & \\
\hline
\end{tabular}

PFF, postoperative periprosthetic fracture.

\section{Figures}




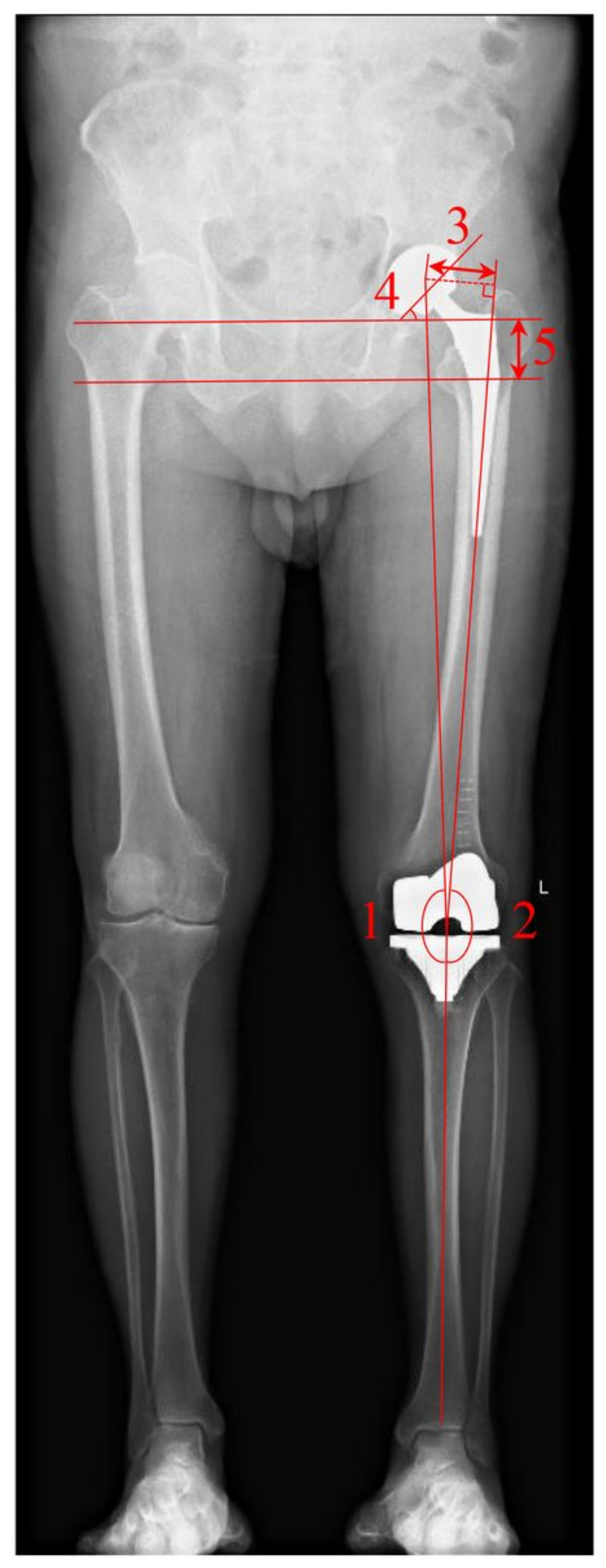

\section{Figure 1}

Radiological angles. 1 Hip-knee-ankle angle (HKA). 2 Femorotibial angle (FTA). 3 Femoral offset (FO). 4 Cup inclination angle. 5 Limb length discrepancy (LLD) 
A

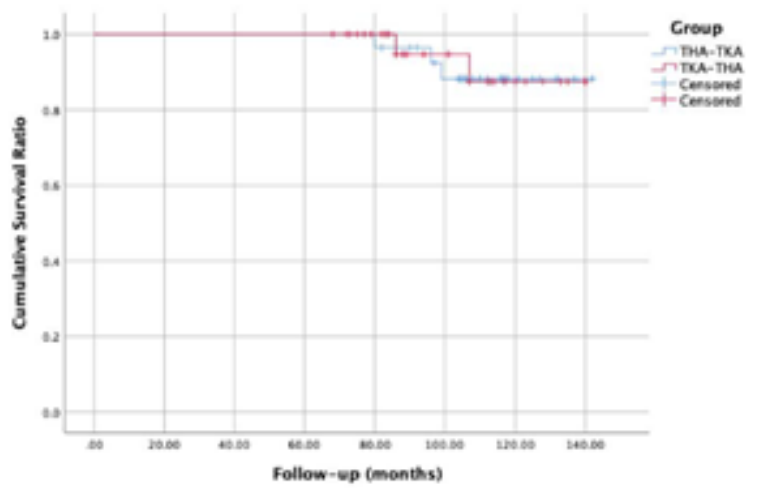

B

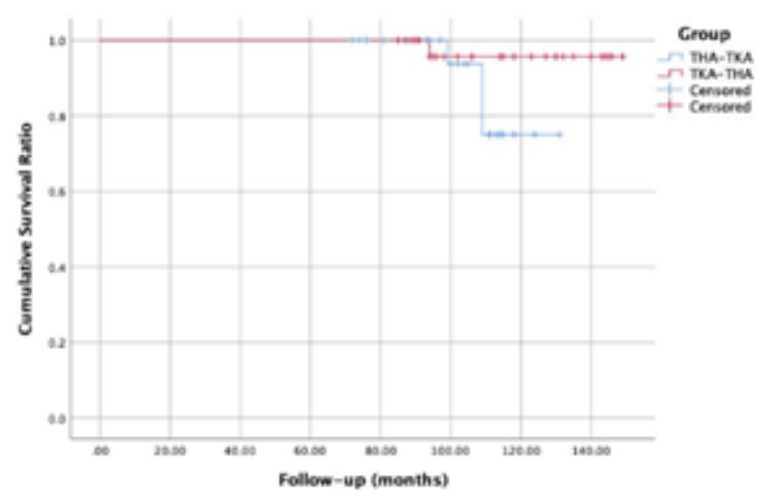

Figure 2

Kaplan-Meier survival curve with revision of THA (A) and TKA (B) for any reason other than infection as the endpoint 


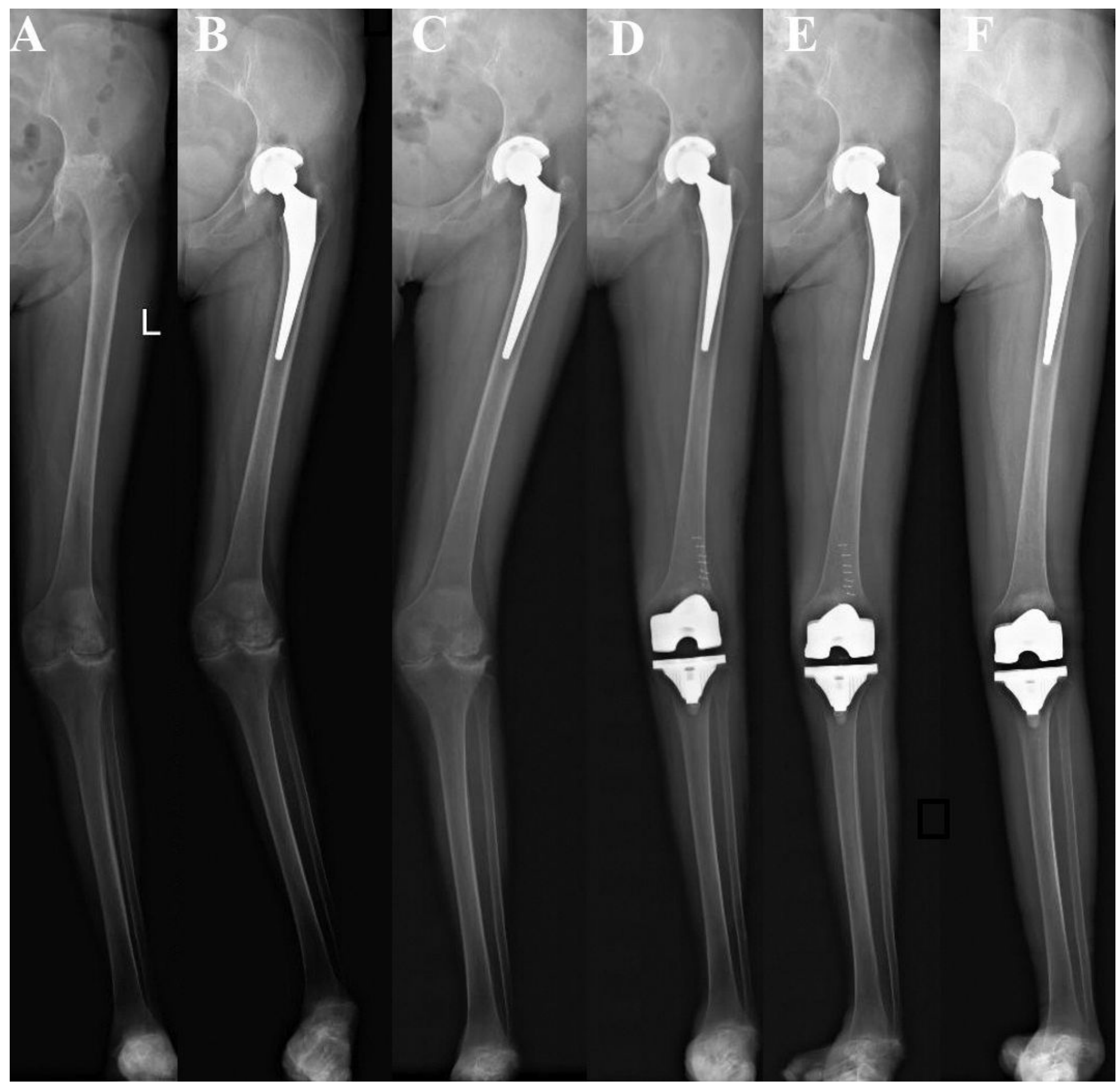

Figure 3

A 43-year-old female with rheumatoid arthritis receiving TKA subsequent to ipsilateral THA. X-ray radiography at preoperative (A), postoperative image of THA (B), and 3-month follow-up (C). Seven months after THA, the patient received ipsilateral TKA. The postoperative radiograph of the lower extremities (D) and a 15-day postoperative radiograph (E) showed stable implant fixation. Postoperative radiographic view $(F)$ at 7-year follow-up demonstrated that the acetabular, femoral, and tibial components were considered to be stable 


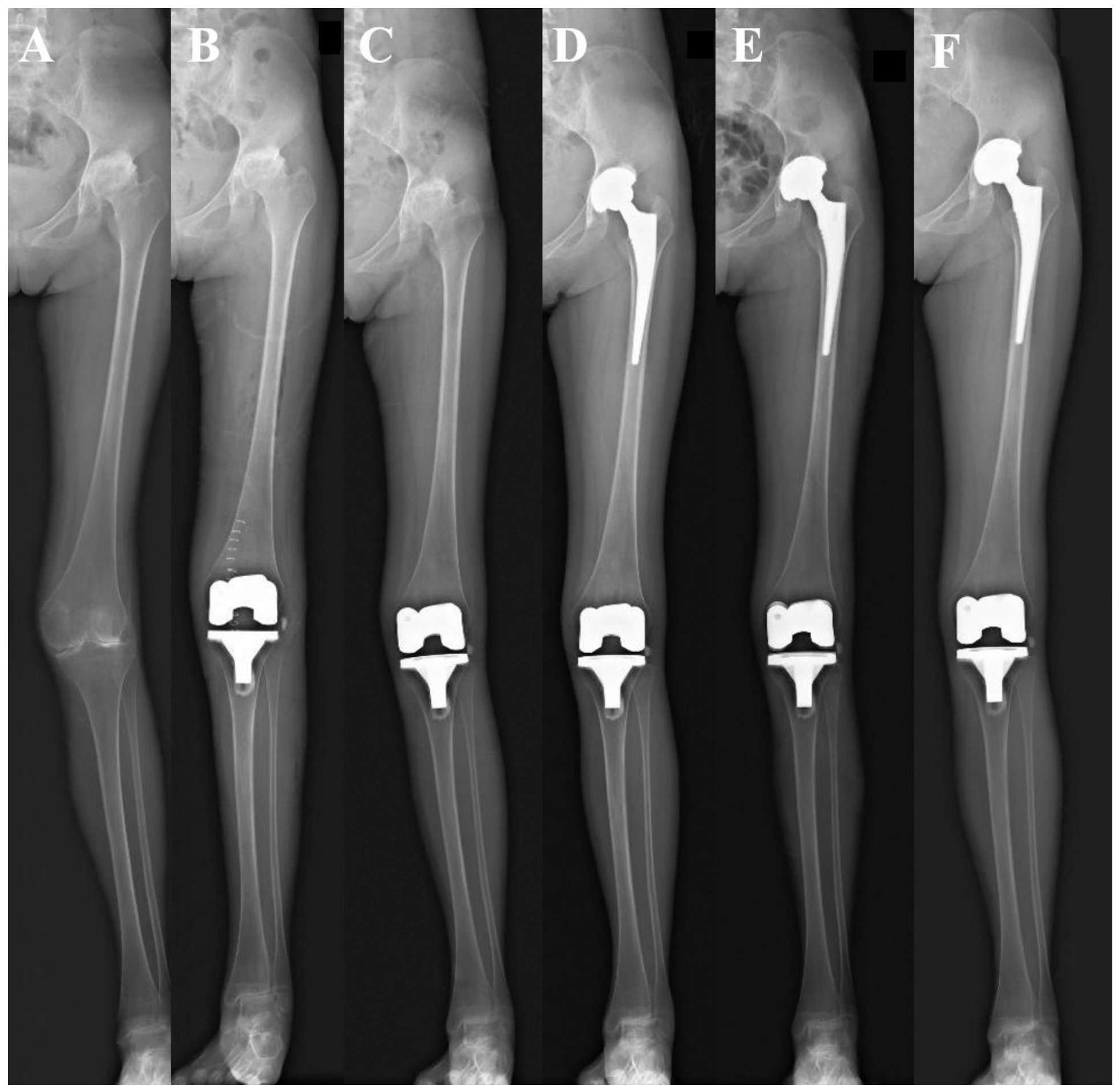

\section{Figure 4}

Radiographs (A-F) of a 27-y-old woman who underwent THA subsequent to ipsilateral TKA for rheumatoid arthritis. (A) Preoperative lower extremity image. (B) Postoperative radiograph of the patient receiving unilateral TKA. (C) Postoperative radiographic view at the 6-month follow-up. (D) One year after prior THA, the patient received ipsilateral THA. The anteroposterior postoperative image of the lower extremities. (E) A 6-month radiograph after subsequent THA. (F) Postoperative radiographic view of acetabular, femoral, and tibial components showed stable implant fixation 